\title{
openheart Implantable loop recorders in patients with heart disease: comparison between patients with and without syncope
}

\author{
Amira Assaf (D) , ${ }^{1}$ Rafi Sakhi, ${ }^{1}$ Michelle Michels, ${ }^{1}$ Jolien W Roos-Hesselink (D) , \\ Judith M A Verhagen, ${ }^{2}$ Rohit E Bhagwandien, ${ }^{1}$ Tamas Szili-Torok, ${ }^{1}$ \\ Dominic Theuns, ${ }^{1}$ Sing-Chien Yap (1) ${ }^{1}$
}

\begin{abstract}
- Additional supplemental material is published online only. To view, please visit the journal online (http://dx.doi.org/10. 1136/openhrt-2021-001748).

To cite: Assaf A, Sakhi R, Michels M, et al. Implantable loop recorders in patients with heart disease: comparison between patients with and without syncope. Open Heart 2021;8:e001748. doi:10.1136/ openhrt-2021-001748
\end{abstract}

Received 5 June 2021 Accepted 22 July 2021

A Check for updates

C) Author(s) (or their employer(s)) 2021. Re-use permitted under CC BY-NC. No commercial re-use. See rights and permissions. Published by BMJ.

${ }^{1}$ Department of Cardiology, Erasmus MC, University Medical Center Rotterdam, Rotterdam, The Netherlands

${ }^{2}$ Department of Clinical

Genetics, University Medical Center Rotterdam, Rotterdam, The Netherlands

Correspondence to Dr Sing-Chien Yap; s.c.yap@ erasmusmc.nl

\section{ABSTRACT}

Objective Patients with heart disease are at increased risk for sudden cardiac death. Guidelines recommend an implantable loop recorder (ILR) for symptomatic patients when symptoms are sporadic and possibly arrhythmiarelated. In clinical practice, an ILR is mainly used in patients with unexplained syncope. We aimed to compare the clinical value of an ILR in patients with heart disease and a history of syncope versus those with non-syncopal symptoms.

Methods In this observational single-centre study, we included symptomatic patients with heart disease who received an ILR. The primary endpoint was an actionable event which was defined as an arrhythmic event leading to a change in clinical management. The secondary endpoint was an event leading to device implantation.

Results One hundred and twenty patients (mean age $47 \pm 17$ years, $49 \%$ men) were included. The underlying disease substrate was inherited cardiomyopathy (31\%), congenital heart disease (28\%), channelopathy (23\%) and other (18\%). Group A consisted of 43 patients with prior syncope and group B consisted of 77 patients with palpitations and/or near-syncope. The median followup duration was 19 months (IQR 8-36). The 3-year cumulative event rate was similar between groups with regard to the primary endpoint (38\% vs $39 \%$ for group $A$ and $B$, respectively, logrank $p=0.54$ ). There was also no difference in the 3-year cumulative rate of device implantation ( $21 \%$ vs $13 \%$ for group $A$ and B, respectively, logrank $\mathrm{p}=0.65)$.

Conclusion In symptomatic patients with heart disease, there is no difference in the yield of an ILR in patients presenting with or without syncope.

\section{INTRODUCTION}

A history of syncope in patients with structural or electrical heart disease is associated with an increased risk of sudden cardiac death (SCD).$^{1-7}$ In this population, the use of an implantable loop recorder (ILR) is recommended to clarify the mechanism of unexplained syncope when there is no indication for an implantable cardioverterdefibrillator (ICD). ${ }^{8}$ An ILR is also recommended in patients who have non-syncopal

\section{Key questions}

What is already known about this subject?

- Suspected arrhythmia-related symptoms in patients with structural or electrical heart disease may be worrisome. When a symptom-rhythm correlation cannot be established with conventional methods, an implantable loop recorder (ILR) may elucidate the underlying arrhythmia. However, in daily practice, ILRs are mainly used in patients with unexplained syncope.

What does this study add?

- In this observational cohort, we compared the actionable event rate in syncopal and non-syncopal patients with heart disease. We found no difference in the actionable event rate and the rate of device implantation between groups. Thus, the yield of an ILR seems similar in patients presenting with syncope or non-syncopal arrhythmia-related symptoms. This study is the first to our knowledge to investigate the yield of an ILR in a large cohort of patients with structural or electrical heart disease.

How might this impact on clinical practice?

- Our results support the use of an ILR in patients with heart disease presenting with suspected arrhythmia-related symptoms, either syncopal or not, when a symptom-rhythm correlation cannot be established by conventional methods.

suspected arrhythmia-related symptoms (ie, palpitations and/or near-syncope), when a symptom-rhythm correlation cannot be established by conventional diagnostic methods. ${ }^{12}$ In clinical practice, however, the use of an ILR for patients with syncope seems to be more common than in those with sporadic nonsyncopal suspected arrhythmia-related symptoms. This is not surprising considering that unexplained syncope is an established risk factor for life-threatening events while symptoms such as palpitations and near-syncope are less specific for serious arrhythmias. We have previously reported our experience with 
ILRs in different study populations, including those with structural and electrical heart disease. ${ }^{9-12}$ However, there is limited data on the yield of an ILR based on the presence or absence of a history of syncope ${ }^{13}{ }^{14}$ Furthermore, limited studies have focused on patients with structural or electrical heart disease. The aim of the present study is to compare the actionable event rate between symptomatic patients with a structural or electrical heart disease who present with and without a history of syncope. Furthermore, we evaluated the rate of pacemaker or ICD implantation stratified by the presence or absence of a history of syncope.

\section{METHODS \\ Study population}

We retrospectively evaluated all symptomatic patients with a structural or electrical heart disease who received an ILR between July 2014 and December 2020 at our institution. These patients were identified using our prospective ILR registry (Early Detection of Ventricular Arrhythmias registry). The indication for the ILR was established in a Heart Team consisting of at least one cardiac electrophysiologist and the treating physician. No patient received an ILR after cryptogenic stroke.

\section{Implantation procedure, device programming and follow-up}

Implantation was done as recommended by the manufacturer using the incision and insertion tool. The device was implanted subcutaneously over the fourth intercostal space on the left haemithorax, either $45^{\circ}$ or $90^{\circ}$ relative to the sternal border. The incision was usually closed with one absorbable suture. After implantation, the patient received the remote monitoring device, as well as instructions about its use for nightly automated transmissions. Patients were discharged on the same day of implantation. The ILR was programmed according to local settings, unless otherwise required. An overview of the implanted devices and nominal settings is provided in online supplemental appendix 1. Patients visited the outpatient clinic at 10 days for wound control and device interrogation. All patients had remote monitoring and had regular ILR interrogation every 6 months. Furthermore, patients had regular follow-up by their treating physician.

\section{Classification of endpoints}

For the current study, patients were divided into two groups. The first group consisted of patients with a history of syncope (group A) and the second group consisted of patients with non-syncopal suspected arrhythmia-related symptoms (group B). The following symptoms were considered non-syncopal suspected arrhythmia-related symptoms: palpitations and/or near-syncope. All patientactivated episodes and automatically detected episodes were screened for actionable events. The primary endpoint of this study was an actionable event which was defined as an arrhythmic event leading to a change in clinical management. The secondary endpoint was an actionable event leading to a pacemaker or ICD implantation.

\section{Statistical analysis}

Continuous data are presented as mean $\pm \mathrm{SD}$ or as median with IQR, as appropriate. Categorical variables are presented by frequencies and percentages. Differences of continuous variables between groups were analysed with a Student's t-test. Differences between categorical variables were analysed with the $\chi^{2}$ test or Fisher's exact test. Event rates were estimated with the Kaplan-Meier method, and differences were compared with logrank. A $p$ value $<0.05$ was considered statistically significant. To correct for differences in baseline variables between groups, multivariate Cox proportional hazards method was used adjusting for age and underlying heart disease. The validity of the assumption of proportionality was verified by visual comparison of Cox and Kaplan-Meier curves, by analysis of interaction with time and by a partial residuals plot. Statistical analyses were performed using SPSS V.21.

\section{RESULTS \\ Study population}

A total of 120 patients were included in the study. Baseline characteristics are presented in table 1 . The average age at ILR implantation was $47 \pm 17$ years and $49 \%$ of patients were men. The underlying disease substrate was inherited cardiomyopathy $(31 \%)$, congenital heart disease (28\%), channelopathy (23\%) and other structural heart disease $(18 \%)$. Other structural heart disease included: ischaemic cardiomyopathy $(\mathrm{n}=10)$, myocarditis $(\mathrm{n}=2)$ and cardiac sarcoidosis $(n=9)$. There were 43 patients with a history of syncope (group A) and 77 patients with non-syncopal symptoms (group B). Group A comprised more patients with other structural heart disease than group B. Group B had a higher proportion of patients with congenital heart disease, prior Holter monitoring and prior documented non-sustained ventricular tachycardia (NSVT).

\section{Primary endpoint}

During a median follow-up of 19 months (IQR 8-36), the primary endpoint was reached in 14 patients $(33 \%)$ of group A and in 28 patients $(36 \%)$ of group B ( $\mathrm{p}=0.68)$. Figure 1 gives an overview of actionable events and corresponding clinical management. Details on patient level are presented in online supplemental appendix 2. The 3 -year cumulative event rate was similar between groups with regard to the primary endpoint (38\% vs $39 \%$ for group $\mathrm{A}$ and $\mathrm{B}$, respectively, logrank $\mathrm{p}=0.54$ ) (figure $2 \mathrm{~A}$ ). The most common actionable events were NSVT $(n=14$, $12 \%)$, supraventricular tachycardia $(\mathrm{n}=11,9 \%)$ and atrial fibrillation (AF) $(\mathrm{n}=8,7 \%)$ (table 2). Group A had a higher proportion of patients with ILR-detected atrioventricular block in comparison to group B ( $7 \%$ vs $0 \%$, $\mathrm{p}=0.04)$. With regard to $\mathrm{AF}$ management, one patient in group $\mathrm{A}$ and two patients in group $\mathrm{B}$ underwent $\mathrm{AF}$ 


\begin{tabular}{|c|c|c|c|}
\hline & $\begin{array}{l}\text { Group A } \\
(n=43)\end{array}$ & $\begin{array}{l}\text { Group B } \\
(n=77)\end{array}$ & P value \\
\hline \multicolumn{4}{|l|}{ Variable } \\
\hline Age at implantation, years & $51 \pm 18$ & $44 \pm 15$ & 0.05 \\
\hline Male sex & $20(47 \%)$ & $39(51 \%)$ & 0.66 \\
\hline Inherited cardiomyopathy & $12(28 \%)$ & $25(32 \%)$ & 0.60 \\
\hline Congenital heart disease & $7(16 \%)$ & $27(35 \%)$ & 0.03 \\
\hline Channelopathy & $12(28 \%)$ & $16(21 \%)$ & 0.38 \\
\hline Other structural heart disease & $12(28 \%)$ & $9(12 \%)$ & 0.03 \\
\hline Family history of SCD & $8(19 \%)$ & $7(9 \%)$ & 0.13 \\
\hline Atrial fibrillation & $4(9 \%)$ & $10(13 \%)$ & 0.55 \\
\hline Diabetes mellitus & $1(2 \%)$ & $6(8 \%)$ & 0.42 \\
\hline Renal insufficiency & $1(2 \%)$ & $1(1 \%)$ & 0.59 \\
\hline \multicolumn{4}{|l|}{$\begin{array}{l}\text { Systemic systolic ventricular } \\
\text { function }\end{array}$} \\
\hline $\begin{array}{l}\text { Moderate dysfunction, } 35 \% \text { - } \\
45 \%\end{array}$ & $6(14 \%)$ & $6(8 \%)$ & 0.35 \\
\hline Mild dysfunction, 45\%-55\% & $10(23 \%)$ & $19(25 \%)$ & 0.86 \\
\hline Normal, $>55 \%$ & $27(61 \%)$ & $52(67 \%)$ & 0.60 \\
\hline Patients with Holter & $37(84 \%)$ & $76(98 \%)$ & $<0.01$ \\
\hline NSVT on Holter & $7(16 \%)$ & $29(38 \%)$ & 0.01 \\
\hline \multicolumn{4}{|l|}{ Medication } \\
\hline AAD & $21(49 \%)$ & $44(57 \%)$ & 0.38 \\
\hline Betablocker & $18(85 \%)$ & $37(84 \%)$ & 0.51 \\
\hline Sotalol & $2(10 \%)$ & $5(11 \%)$ & 0.51 \\
\hline Verapamil/diltiazem & $2(10 \%)$ & $2(5 \%)$ & 0.62 \\
\hline AAD class I & $1(5 \%)$ & $1(2 \%)$ & 0.59 \\
\hline Amiodaron & - & $1(2 \%)$ & 0.36 \\
\hline Oral anticoagulation & $6(14 \%)$ & $13(17 \%)$ & 0.67 \\
\hline
\end{tabular}

Data are presented as $n(\%)$ or mean \pm SD.

$A A D$, antiarrhythmic drugs; ILR, implantable loop recorder; LVEF, left ventricular ejection fraction; NSVT, non-sustained ventricular tachycardia; SCD, sudden cardiac death.

ablation. Patients with ILR-detected AF already used oral anticoagulation or did not need oral anticoagulation due to a low thromboembolic risk profile. In the univariate Cox regression analysis, syncope was not associated with actionable events (HR 0.83 (95\% CI 0.44 to 1.56), $\mathrm{p}=0.54)$. After correction for differences in baseline variables using multivariate Cox regression analysis, syncope was also not associated with actionable events (adjusted HR 0.79 (95\% CI 0.41 to 1.54$), \mathrm{p}=0.49$ ).

\section{Secondary endpoint}

The secondary event rate was reached in seven $(16 \%)$ patients of group A and in eight (10\%) patients of group B $(p=0.35)$. The 3-year cumulative rate of device implantation was similar between groups (21\% vs $13 \%$ for group $\mathrm{A}$ and $\mathrm{B}$, respectively, logrank $\mathrm{p}=0.65$ ) (figure $2 \mathrm{~B}$ ). In group A, two patients $(5 \%)$ received an ICD; one after

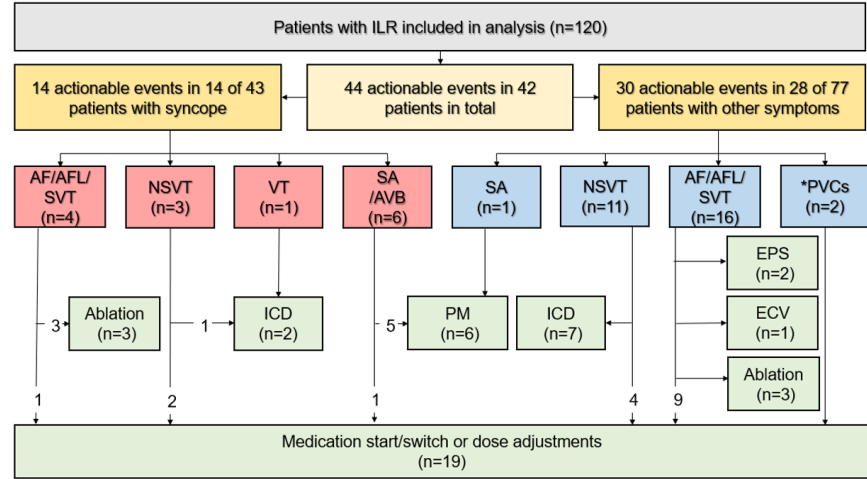

Figure 1 Overview of actionable events and clinical management. *Symptomatic PVCs. AF, atrial fibrillation; AFL, atrial flutter; AVB, atrioventricular block; ECV, electrical cardioversion; EPS, electrophysiology study; ICD, implantable cardioverter defibrillator; ILR, implantable loop recorder; NSVT, non-sustained ventricular tachycardia; PM, pacemaker; PVC, premature ventricular complex; SA, sinus arrest; SVT, supraventricular tachycardia; VT, ventricular tachycardia.

the detection of sustained VT. Furthermore, five patients (12\%) underwent a pacemaker implantation of whom three patients had documented atrioventricular block and two patients had symptomatic sinus arrest. In group B, seven patients $(9 \%)$ received an ICD after the detection of NSVT and one patient $(1 \%)$ underwent a pacemaker implantation for symptomatic sinus arrest. In group A there were more patients who received a pacemaker than in group B $(12 \%$ vs $1 \%, \mathrm{p}=0.01)$. In the univariate Cox regression analysis, syncope was not associated with device implantation (HR 1.26 (95\% CI 0.47 to 3.39), $\mathrm{p}=0.65$ ). After correction for differences in baseline variables using multivariate Cox regression analysis, syncope was also not associated with device implantation (adjusted HR 0.79 (95\% CI 0.27 to 2.33), $\mathrm{p}=0.67$ ).

\section{DISCUSSION}

The purpose of this study was to compare the clinically relevant arrhythmic event rate of an ILR in patients with cardiac disease who have a history of syncope versus those with non-syncopal suspected arrhythmia-related symptoms. We found no difference in the yield of an ILR between groups, either defined as the rate of actionable
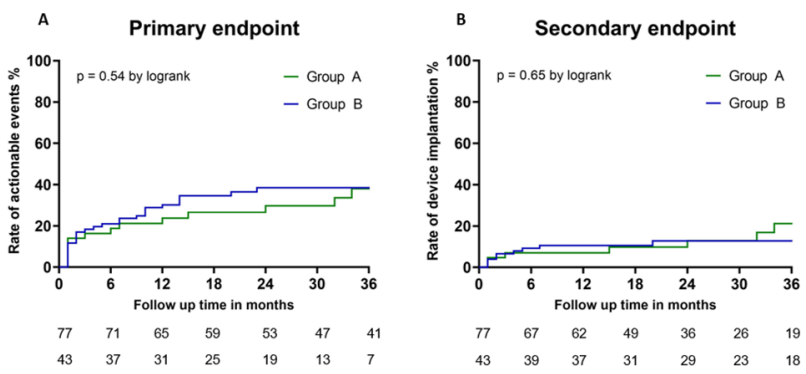

Figure 2 Comparison of the cumulative event rates for actionable events $(A)$ and device implantations $(B)$ between groups. 
Table 2 Comparison of frequency of actionable events between groups

\begin{tabular}{llll}
\hline Type of actionable event & $\begin{array}{l}\text { Group A } \\
(\mathbf{n = 4 3 )}\end{array}$ & $\begin{array}{l}\text { Group B } \\
(\mathbf{n}=77)\end{array}$ & P value \\
\hline NSVT & $3(7 \%)$ & $11(14 \%)$ & 0.23 \\
Supraventricular tachycardia & $3(7 \%)$ & $8(10 \%)$ & 0.74 \\
Atrial fibrillation & $1(2 \%)$ & $7(9 \%)$ & 0.26 \\
\hline Sinus arrest & $3(7 \%)$ & $1(1 \%)$ & 0.13 \\
AV block & $3(7 \%)$ & - & 0.04 \\
PVCs & - & $2(3 \%)$ & 0.54 \\
Atrial flutter & - & $1(1 \%)$ & 1.00 \\
Sustained VT & $1(2 \%)$ & - & 0.36 \\
\hline
\end{tabular}

Data are presented as $n(\%)$.

$\mathrm{AV}$, atrioventricular; NSVT, non-sustained ventricular tachycardia;

PVC, premature ventricular complex; VT, ventricular tachycardia.

events or the rate of device implantations. It should be mentioned that the majority of patients in our study population $(82 \%)$ had a background of either a genetic or congenital heart disease. To our knowledge, this study is the first to investigate the yield of an ILR in a large cohort of adults with structural or electrical heart disease.

\section{Role of ILRs in high-risk patients}

An ILR can be a useful diagnostic tool in selected symptomatic patients with structural or electrical heart disease who are at increased risk of SCD. Continuous arrhythmia monitoring can provide: (1) symptom-rhythm correlation; (2) detection of asymptomatic clinically relevant arrhythmias (eg, AF, NSVT) and (3) patient reassurance. For example, detection of asymptomatic NSVT can be relevant for risk stratification in patients with hypertrophic cardiomyopathy or arrhythmogenic right ventricular cardiomyopathy. ${ }^{215}$ Furthermore, the detection of subclinical AF may necessitate the use of oral anticoagulation in patients with hypertrophic cardiomyopathy. ${ }^{12}$ The current European Society of Cardiology guidelines recommend an ILR for patients with inherited cardiomyopathies or channelopathies who present with recurrent episodes of unexplained syncope and who are at low risk of SCD (class IIa indication). ${ }^{8}$ Furthermore, an ILR is also recommended in patients with infrequent, suspected arrhythmia-related symptoms, if conventional methods fail to provide a symptom-rhythm correlation. ${ }^{12}$ However, data supporting these recommendations in the specific population of patients with structural or electrical heart disease are scarce. ${ }^{9-1216-21}$ The current study provides an overview of the yield of an ILR in this specific population.

\section{Syncope versus other suspected arrhythmia-related symptoms}

In clinical practice, an ILR is mainly used in patients with unexplained syncope. ${ }^{131422}$ This is not surprising considering the unique feature of an ILR to automatically record the rhythm during a syncopal event. For this purpose, conventional Holters or event recorders are less suitable considering the infrequent nature of the events. In contrast, an ILR is less frequently used to elucidate the nature of palpitations, although an older randomised trial has shown that an ILR can be a cost-effective approach in patients with infrequent unexplained palpitations in comparison to a conventional strategy. ${ }^{20}$ Furthermore, a recent large single-centre cohort study from the Mayo Clinic showed that patients who received an ILR for palpitations more often had a change in clinical management than patients who received an ILR for syncope. ${ }^{14}$ Another single-centre cohort study by Smith et al also demonstrated a higher diagnostic yield of the ILR in patients with palpitations in comparison to patients with syncope $(60 \%$ vs $28 \%) .{ }^{13}$ Both studies mainly comprised patients with a structural normal heart. Our study extends these findings to patients with structural and electrical heart disease by demonstrating no difference in actionable events between symptomatic patients with and without syncope.

Although the rate of device implantations was similar between groups in our study, there was, however, a higher incidence of pacemaker implantations in patients with syncope in comparison to patients without a history of syncope. This higher likelihood of a pacemaker implantation in patients with a history of syncope has been shown before in a large administrative database of patients with cardiovascular disease monitored with an ILR. ${ }^{23}$ The above-mentioned findings suggest that an ILR might be useful in a broader selection of patients with suspected arrhythmia-related symptoms (not only those presenting with syncope), and that there is a difference in the type of ILR-detected arrhythmia between those with or without syncope.

\section{ILRs versus wearables}

The increased use of personal wearables, for example, the Apple Watch, has supplemented the field of ambulatory rhythm monitoring. With these devices, it is now possible to actively record a single-lead ECG during suspected arrhythmia-related symptoms. ${ }^{24}{ }^{25}$ Although these devices may elucidate the nature of sustained palpitations, they are less suitable for short-lived arrhythmia-related symptoms and unsuitable for syncopal events and asymptomatic events which may be clinically relevant (eg, NSVT) . The exact role of these wearables in patient management remains to be elucidated.

\section{Study limitations}

We present data from a single academic centre with a highly selective patient population in which the majority had a genetic or congenital heart disease. The indication to implant an ILR was based on a careful multiparametric analysis, including among others, the specific symptoms of the patient, underlying heart disease, conduction abnormalities and estimated risk of SCD. Therefore, the results should not be extrapolated to any symptomatic patient with structural or electrical heart disease. 


\section{Conclusions}

In our study, there was no difference in the actionable event rate in patients with heart disease who have a history of syncope versus those with non-syncopal suspected arrhythmia-related symptoms. Moreover, the rate of pacemaker or ICD implantation was similar between groups. Our results support the use of an ILR in heart disease patients presenting with suspected arrhythmia-related symptoms, either syncopal or not, when a symptomrhythm correlation cannot be established by conventional methods. Further studies are required to refine the role of ILRs in risk stratification in certain patient populations.

Contributors Study design: S-CY. Data collection: AA and RS. Data analysis and statistical analysis: AA, S-CY. Manuscript draft: AA, S-CY. Critical revision, editing and approval of the final manuscript: all authors.

Funding The authors have not declared a specific grant for this research from any funding agency in the public, commercial or not-for-profit sectors.

Competing interests S-CY has received a research grant from Medtronic. Other authors have no relevant affiliations or financial involvement with any organisation or entity with a financial interest or financial conflict with the subject matter or materials discussed in this manuscript.

Patient consent for publication Not required.

Ethics approval All patients gave informed consent for the implantation procedure. The Medical Ethics Committee of the Erasmus MC reviewed the study (MEC-2017-052), and this study was not subjected to the Dutch Medical Research Involving Human Subjects Act. The study was carried out according to the ethical principles for medical research involving human subjects established by the Declaration of Helsinki, protecting the privacy of all the participants and the confidentiality of their personal information. This research was done without patient or public involvement.

Provenance and peer review Not commissioned; externally peer reviewed.

Data availability statement Data are available upon reasonable request. All data relevant to the study are included in the article or uploaded as supplementary information.

Open access This is an open access article distributed in accordance with the Creative Commons Attribution Non Commercial (CC BY-NC 4.0) license, which permits others to distribute, remix, adapt, build upon this work non-commercially, and license their derivative works on different terms, provided the original work is properly cited, appropriate credit is given, any changes made indicated, and the use is non-commercial. See: http://creativecommons.org/licenses/by-nc/4.0/.

\section{ORCID iDs}

Amira Assaf http://orcid.org/0000-0002-3223-1968

Jolien W Roos-Hesselink http://orcid.org/0000-0002-6770-3830

Sing-Chien Yap http://orcid.org/0000-0003-4520-2725

\section{REFERENCES}

1 Al-Khatib SM, Stevenson WG, Ackerman MJ. 2017 AHA/ACC/HRS guideline for management of patients with ventricular arrhythmias and the prevention of sudden cardiac death: a report of the American College of Cardiology/American heart association Task force on clinical practice guidelines and the heart rhythm Society. Circulation 2017.

2 Priori SG, Blomstrom-Lundqvist C, Mazzanti A. ESC guidelines for the management of patients with ventricular arrhythmias and the prevention of sudden cardiac death: the task force for the management of patients with ventricular arrhythmias and the prevention of sudden cardiac death of the European Society of cardiology (ESC). endorsed by: association for European paediatric and congenital cardiology (AEPC). Eur Heart $J$ 2015;2015:2793-867.

3 Liu JF, Jons C, Moss AJ, et al. Risk factors for recurrent syncope and subsequent fatal or near-fatal events in children and adolescents with long QT syndrome. J Am Coll Cardiol 2011;57:941-50.
4 Probst V, Veltmann C, Eckardt L, et al. Long-term prognosis of patients diagnosed with Brugada syndrome: results from the FINGER Brugada syndrome registry. Circulation 2010;121:635-43.

5 Spirito P, Autore C, Rapezzi C, et al. Syncope and risk of sudden death in hypertrophic cardiomyopathy. Circulation 2009;119:1703-10.

6 Olde Nordkamp LRA, Vink AS, Wilde AAM, et al. Syncope in Brugada syndrome: prevalence, clinical significance, and clues from history taking to distinguish arrhythmic from nonarrhythmic causes. Heart Rhythm 2015;12:367-75.

7 Corrado D, Calkins H, Link MS, et al. Prophylactic implantable defibrillator in patients with arrhythmogenic right ventricular cardiomyopathy/dysplasia and no prior ventricular fibrillation or sustained ventricular tachycardia. Circulation 2010;122:1144-52.

8 Brignole M, Moya A, de Lange FJ. ESC guidelines for the diagnosis and management of syncope. Eur Heart J 2018;2018:1883-948.

9 Sakhi R, Assaf A, Theuns DAMJ, et al. Outcome of Insertable cardiac monitors in symptomatic patients with Brugada syndrome at low risk of sudden cardiac death. Cardiology 2020;145:413-20.

10 Sakhi R, Kauling RM, Theuns DA, et al. Early detection of ventricular arrhythmias in adults with congenital heart disease using an insertable cardiac monitor (EDVA-CHD study). Int J Cardiol 2020;305:63-9.

11 Sakhi R, Theuns DAMJ, Bhagwandien RE, et al. Value of implantable loop recorders in patients with structural or electrical heart disease. $J$ Interv Card Electrophysiol 2018;52:203-8.

12 Sakhi R, Huurman R, Theuns DAMJ, et al. Incremental value of an insertable cardiac monitor in patients with hypertrophic cardiomyopathy with low or intermediate risk for sudden cardiac death. Cardiology 2021;146:207-12.

13 Smith A, Perdue M, Vojnika J. The diagnostic yield of implantable loop recorders stratified by indication: 'real-world' use in a large academic hospital. J Interv Card Electrophysiol 2020 doi:10.1007/ s10840-020-00815-w

14 Padmanabhan D, Kancharla K, El-Harasis MA, et al. Diagnostic and therapeutic value of implantable loop recorder: a tertiary care center experience. Pacing Clin Electrophysiol 2019;42:38-45.

15 Elliott PM, Anastasakis A, Borger MA. ESC guidelines on diagnosis and management of hypertrophic cardiomyopathy: the task force for the diagnosis and management of hypertrophic cardiomyopathy of the European Society of cardiology (ESC). Eur Heart $J$ 2014;2014:2733-79.

16 Dodeja AK, Thomas C, Daniels CJ, et al. Detection of arrhythmias in adult congenital heart disease patients with LINQ ${ }^{\mathrm{TM}}$ implantable loop recorder. Congenit Heart Dis 2019;14:745-51.

17 Bezzerides VJ, Walsh A, Martuscello M, et al. The real-world utility of the LINQ implantable loop recorder in pediatric and adult congenital heart patients. J Am Coll Cardiol EP 2019;5:245-51.

18 Mason PK, Wood MA, Reese DB, et al. Usefulness of implantable loop recorders in office-based practice for evaluation of syncope in patients with and without structural heart disease. Am J Cardiol 2003:92:1127-9.

19 Solano A, Menozzi C, Maggi R, et al. Incidence, diagnostic yield and safety of the implantable loop-recorder to detect the mechanism of syncope in patients with and without structural heart disease. Eur Heart J 2004;25:1116-9.

20 Giada F, Gulizia M, Francese M, et al. Recurrent unexplained palpitations (RUP) study comparison of implantable loop recorder versus conventional diagnostic strategy. J Am Coll Cardiol 2007:49:1951-6.

21 Pezawas T, Stix G, Kastner J, et al. Implantable loop recorder in unexplained syncope: classification, mechanism, transient loss of consciousness and role of major depressive disorder in patients with and without structural heart disease. Heart 2008;94:e17.

22 Bezzerides VJ, Walsh A, Martuscello M, et al. The real-world utility of the LINQ implantable loop recorder in pediatric and adult congenital heart patients. JACC Clin Electrophysiol 2019;5:245-51.

23 Mittal S, Rogers J, Sarkar S, et al. Real-world incidence of pacemaker and defibrillator implantation following diagnostic monitoring with an Insertable cardiac monitor. Am J Cardiol 2019;123:1967-71.

24 Burke J, Haigney MCP, Borne R, et al. Smartwatch detection of ventricular tachycardia: case series. HeartRhythm Case Rep 2020;6:800-4.

25 Perez MV, Mahaffey KW, Hedlin H, et al. Large-scale assessment of a smartwatch to identify atrial fibrillation. N Engl J Med 2019;381:1909-17. 